\title{
Rapid detection of porins by matrix-assisted laser desorption/ionization-time of flight mass spectrometry
}

\author{
Yan-Yan Hu, Jia-Chang Cai, Hong-Wei Zhou, Rong Zhang and Gong-Xiang Chen* \\ Second Affiliated Hospital of Zhejiang University, Zhejiang University, Hangzhou, China
}

OPEN ACCESS

Edited by:

Kunihiko Nishino,

Osaka University, Japan

Reviewed by:

Henrietta Venter,

University of South Australia, Australia

Ying Zhou,

AmberGen Inc., USA

${ }^{*}$ Correspondence:

Gong-Xiang Chen,

Second Affiliated Hospital of Zhejiang

University, Zhejiang University,

88 Jiefang Road, Hangzhou 310009,

China

chengong218@yeah.net

Specialty section:

This article was submitted to

Antimicrobials, Resistance

and Chemotherapy,

a section of the journal

Frontiers in Microbiology

Received: 24 May 2015

Accepted: 17 July 2015

Published: 04 August 2015

Citation:

Hu Y-Y, Cai J-C, Zhou H-W, Zhang $R$ and Chen G-X (2015) Rapid detection of porins by matrix-assisted laser desorption/ionization-time of flight mass spectrometry.

Front. Microbiol. 6:784. doi: 10.3389/fmicb.2015.00784
The rapid and cost-efficient determination of carbapenem resistance is an important prerequisite for the choice of an adequate antibiotic therapy. A MALDI-TOF MS-based assay was set up to detect porins in the current study. A loss of the components of porin alone such as OmpK35/OmpK36 or together with the production of carbapenemases will augment the carbapenem resistance. Ten strains of Escherichia coli and eight strains of Klebsiella pneumoniae were conducted for both sodium dodecylsulfatepolyacrylamide gel electrophoresis (SDS-PAGE) and MALDI-TOF MS analysis. MALDITOF/TOF MS analysis was then performed to verify the correspondence of proteins between SDS-PAGE and MALDI-TOF MS. The results indicated that the mass spectrum of ca. 35,000, 37,000, and 38,000-m/z peaks of E. coli ATCC 25922 corresponded to OmpA, OmpC, and OmpF with molecular weight of approximately ca. 38, 40, and 41 $\mathrm{kDa}$ in SDS-PAGE gel, respectively. The band of OmpC and OmpF porins were unable to be distinguished by SDS-PAGE, whereas it was easy to be differentiated by MALDI-TOF MS. As for K. pneumoniae isolates, the mass spectrum of ca. 36,000 and 38,600$\mathrm{m} / \mathrm{z}$ peaks was observed corresponding to OmpA and OmpK36 with molecular weight of approximately ca. 40 and $42 \mathrm{kDa}$ in SDS-PAGE gel, respectively. Porin OmpK35 was not observed in the current SDS-PAGE, while a $37,000-\mathrm{m} / \mathrm{z}$ peak was found in K. pneumoniae ATCC 13883 and carbapenem-susceptible strains by MALDI-TOF MS which was presumed to be the characteristic peak of the OmpK35 porin. Compared with SDS-PAGE, MALDI-TOF MS is able to rapidly identify the porin-deficient strains within half an hour with better sensitivity, less cost, and is easier to operate and has less interference.

Keywords: outer membrane protein, MALDI-TOF MS, SDS-PAGE, E. coli, K. pneumoniae

\section{Introduction}

Carbapenems, which are effective against extended spectrum $\beta$-Lactamases (ESBLs) or AmpCproducing strains, are considered to be the most active antibiotics against ESBLs-producing Enterobacteriaceae and are thus extensively used for the treatment of serious infections especially the infections caused by multi-drug resistant Enterobacteriaceae (Baldwin et al., 2008). However, abusing of antibiotics is becoming an increasingly serious problem which followed by emergence 
of carbapenem resistance. Thus, a rapid detection and determination method of the drug resistance mechanism is urgently needed in response to the growing number of carbapenem-resistant Enterobacteriaceae. According to the reports (Queenan and Bush, 2007; Liu et al., 2008; PappWallace et al., 2011; Nagaraj et al., 2012), the predominant carbapenem resistant mechanisms in Enterobacteriaceae are attributed to the following two aspects: (1) production of carbapenemases; (2) combination of high-level production of ESBLs or AmpC $\beta$-lactamases together with loss of porin. Porins allow carbapenems to diffuse into the bacterial, thus, missing or decreased expression of porins lead to carbapenem resistance against bacterial. The most relevant porins with carbapenem-resistance are OmpC and OmpF in Escherichia coli and OmpK35 and OmpK36 in Klebsiella pneumoniae (Nikaido et al., 1983; Alberti et al., 1995; Domenech-Sanchez et al., 2003).

Matrix-assisted laser desorption/ionization time of flight mass spectrometry (MALDI-TOF MS), a recently introduced technique for microorganism identification (Bizzini and Greub, 2010; Wieser et al., 2012) has also been applied for the rapid detection of antibiotic susceptibility. MALDI-TOF MS-based assay for the detection of $\beta$-lactamases especially carbapenemases activity had been described (Burckhardt and Zimmermann, 2011; Hrabak et al., 2011; Sparbier et al., 2012). $\beta$-lactamases producing strains can be rapidly detected through comparing the characteristic peaks of $\beta$-lactam antibiotics with peaks after incubation of antibiotics together with bacteria. The characteristic spectral peaks of antibiotics would disappear if the bacteria produce $\beta$-lactamases. The average turn-around time of the method is approximately $3 \sim 4 \mathrm{~h}$, much faster than Modified Hodge Test (MHT) which is recommend by CLSI for carbapenem detection and takes for about $16 \sim 18 \mathrm{~h}$ (CLSI, 2014). Besides this, MALDI-TOF MS had been used in rapid detection of other drug-resistance such as methicillin resistant Staphylococcus aureus (Edwards-Jones et al., 2000), vancomycinresistant Enterococcus spp. (Griffin et al., 2012) and rifampin or isoniazid resistant Mycobacterium tuberculosis (Ikryannikova et al., 2007). Though the MALDI-TOF MS has been successfully used in detection of antibiotic-resistance, few reports of rapid detection of porins was reported. Though LC-MS/MS (Prajanban et al., 2012) or even LC-MALDI MS (Liu et al., 2011) were already used for protein identification and characterization in analytical chemistry, these devices cost much higher than MALDI-TOF MS and were only limited for laboratory research not for microorganism identification. Porins are usually detected using the classical sodium dodecylsulfate-polyacrylamide gel electrophoresis (SDS-PAGE) method, and it is laborious, and time-consuming. Therefore, we have applied the method for the rapid detection of porins in K. pneumoniae (Cai et al., 2012).

In the current study, analysis of porins from 18 isolates including 10 strains of E. coli and 8 strains of K. pneumoniae were conducted by MALDI-TOF MS. MALDI-TOF/TOF MS was then performed to identify the bands in the SDSPAGE gel and correlate them with the proteins observed in MS.

\section{Materials and Methods}

\section{Bacterial Strains}

A total of 18 non-duplicated Enterobacteriaceae strains including one carbapenem susceptible E. coli isolate, ATCC 25922, eight E. coli isolates with carbapenem resistance or reduced susceptibility, one carbapenem susceptible K. pneumoniae isolate, ATCC 13883, and six K. pneumoniae isolates with carbapenem resistance or reduced susceptibility were selected in this study (Table 1). Species identification for the 18 isolates were initially performed with the Vitek 2 compact system (bioMérieux, Durham, NC, USA) and then confirmed by MALDI-TOF MS (Bruker Daltonik GmbH, Bremen, Germany; MALDI Biotyper 3.0).

\section{Antimicrobial Susceptibility Testing}

The minimum inhibitory concentrations (MICs) of imipenem, meropenem, and ertapenem were determined by Mueller-Hinton (M-H) agar dilution method and were interpreted in accordance with the standards of Clinical Laboratory Standards Institute (CLSI; CLSI, 2013), E. coli ATCC 25922 and K. pneumoniae ATCC 13883 were used for quality control.

\section{PCR Amplification}

Screening for common ESBLs or carbapenemase genes, including bla $a_{\mathrm{TEM}}$ (Yu et al., 2007), bla $a_{\mathrm{SHV}}$ (Yu et al., 2007), bla $a_{\mathrm{CTX}-\mathrm{M}}$ (Yu et al., 2007), AmpC (Perez-Perez and Hanson, 2002), bla $a_{\mathrm{KPC}}$ (Yigit et al., 2001), bla $a_{\mathrm{IMP}}$ (Queenan and Bush, 2007), bla $a_{\mathrm{VIM}}$ (Queenan and Bush, 2007), and bla $a_{\mathrm{NDM}}$ (Zhang et al., 2013) were performed by PCR amplification using specific primers in a Tpersonal Cycler (Biometra, Germany). The PCR products were then sequenced using an ABI3730 Sequencer (Applied Biosystems, Foster City, CA, USA), and compared with the reported sequences from GenBank.

\section{Analysis of Outer Membrane Proteins (OMPs)}

Outer membrane proteins (OMPs) were extracted as described previously (Hernandez-Alles et al., 1999). OMPs were solubilized in electrophoresis sample buffer and boiled for $5 \mathrm{~min}$ before electrophoretic analysis. Strains were grown overnight in nutrient broth without $\mathrm{NaCl}$ at $37^{\circ} \mathrm{C}$ with shaking. OMPs were determined by SDS-PAGE with a $5 \%$ stacking gel $(4.86 \%$ acrylamide $/ 0.17 \%$ bisacrylamide $/ 0.1 \%$ SDS) and a $15 \%$ separating gel (14.5\% acrylamide/ $0.5 \%$ bisacrylamide/ $0.1 \%$ SDS) containing $20 \%$ urea. The $0.75 \mathrm{~mm}$ thickness gel was run at a constant current of $20 \mathrm{~mA}$ for $140 \mathrm{~min}$ with a Mini Protein 3 slab electrophoresis cell (Bio-Rad). OMPs were detected with Coomassie brilliant blue R-250 staining.

\section{MALDI-TOF MS Analysis}

One microliter of the extracted OMPs was applied onto a polished steel 96-spot target (Bruker Daltonik $\mathrm{GmbH}$, catalog: 224989) and after drying at room temperature, $1 \mu \mathrm{l}$ of $20 \mathrm{mg} / \mathrm{ml}$ 2,5-dihydroxybenzoic acid matrix (DHB, dissolved in 50\% $\mathrm{ACN}+2.5 \% \mathrm{TFA}$ ) was added to each target spot. MALDI-TOF MS was performed with the flexControl 3.3 software (Bruker Daltonik $\mathrm{GmbH}$ ) operating in positive linear ion mode between 
TABLE 1 | Carbapenem susceptibility and carbapenemase and $\beta$-lactamase genes of the selected 18 strains.

\begin{tabular}{|c|c|c|c|c|c|c|}
\hline Strain* & Previous name & $\beta$-lactamases & $\mathrm{IPM}^{\#}(\mu \mathrm{g} / \mathrm{ml})$ & MEM $(\mu \mathrm{g} / \mathrm{ml})$ & ETP $(\mu \mathrm{g} / \mathrm{ml})$ & Reference \\
\hline EC1 & - & CMY-2, CTX-M-14, TEM-1 & 16 & 16 & 32 & This study \\
\hline EC2 & E21 & KPC-2, TEM-1 & 16 & 16 & 32 & Cai et al. (2014) \\
\hline EC3 & - & CTX-M-14 & 1 & 2 & 8 & This study \\
\hline EC4 & E6 & KPC-2, CTX-M-15 & 8 & 16 & 64 & Cai et al. (2014) \\
\hline EC5 & E1 & KPC-2, CTX-M-15 & 2 & 2 & 8 & Cai et al. (2014) \\
\hline EC6 & - & TEM-1, CTX-M-55, CMY-2 & 2 & 1 & 8 & This study \\
\hline EC7 & - & TEM-1, CTX-M-15, CMY-2 & 16 & 32 & 128 & This study \\
\hline EC8 & - & TEM-1, CTX-M-14, DHA-1, SHV-12 & 8 & 8 & 16 & This study \\
\hline EC9 & - & - & $\leq 0.125$ & $\leq 0.125$ & $\leq 0.125$ & This study \\
\hline KP1 & Z2110 & TEM-1, SHV-11, CTX-M-14, DHA-1 & 4 & 2 & 32 & Cai et al. (2012) \\
\hline KP2 & Z2554 & TEM-1, SHV-11, CTX-M-14 & 0.5 & 8 & 16 & Cai et al. (2012) \\
\hline KP3 & Z4 & IMP-4, TEM-1, SHV-1 & 32 & 32 & 256 & Cai et al. (2012) \\
\hline KP4 & Z5 & IMP-4, TEM-1, SHV-12 & 1 & 1 & 2 & Cai et al. (2012) \\
\hline KP5 & $\mathrm{K} 1$ & KPC-2, TEM-1, SHV-11, CTX-M-14 & 4 & 4 & 8 & Cai et al. (2012) \\
\hline KP6 & K10 & KPC-2, TEM-1, SHV-12, CTX-M-14 & 128 & 256 & $>256$ & Cai et al. (2012) \\
\hline KP7 & S1 & - & $\leq 0.125$ & $\leq 0.125$ & $\leq 0.125$ & Cai et al. (2012) \\
\hline ATCC 25922 & - & - & $\leq 0.125$ & $\leq 0.125$ & $\leq 0.125$ & This study \\
\hline ATCC 13883 & - & - & $\leq 0.125$ & $\leq 0.125$ & $\leq 0.125$ & This study \\
\hline
\end{tabular}

*EC1-EC8, clinically isolated E. coli strains with carbapenem resistance or reduced susceptibility; EC9, clinically isolated E. coli strain with carbapenem-susceptible; KP1-KP6, clinically isolated K. pneumoniae strains with carbapenem-resistance or reduced susceptibility; KP7, clinically isolated K. pneumoniae strain with carbapenemsusceptible.

\#IPM, imipenem; MEM, meropenem; ETP, ertapenem.

5,000 and 50,000 Da. The protocol used in this study had a slight modification on LP_66KDa.par (provided by the manufacturer). The main parameters were set as follows: mode, low range; ion source 1, $19.00 \mathrm{kV}$; ion source 2, $17.20 \mathrm{kV}$; lens, $6.00 \mathrm{kV}$; pulsed ion extraction, $0 \mathrm{~ns}$; digitizer trigger level, $1000 \mathrm{mV}$; laser range $30 \%$, laser offset $15-30 \%$, laser frequency, $60 \mathrm{~Hz}$; linear 13.0X, Sample rate 2.0; electronic gain: enhanced $100 \mathrm{mV}$; shots: 100, and 500 shots were summed up for each sample. The protein calibration standard II (Bruker Daltonik $\mathrm{GmbH}$ ) with the significant peaks of ca. 22,307.0, 23,982.0, 33,216.0, $44,613.0,66,431.0,89,225.0$, and $132,861.0 \mathrm{~m} / \mathrm{z}$ Dalton was used for calibration, and the calibration error was regulated under 300 ppm strictly. The mass spectra were analyzed using Bruker daltonics flexAnalysis 3.3 software.

\section{Tryptic in-Gel Digestion and MALDI-TOF/TOF MS Analysis}

The OMP bands were carefully excised and transferred into a $1.5 \mathrm{ml}$ Eppendorf centrifuge tube. Gel chips were destained with $30 \mu \mathrm{M} \mathrm{NH} \mathrm{NHCO}_{3} / 30 \%$ ACN. After freeze-drying, the gels were hydrated in $5 \mu \mathrm{L}(2.5 \mathrm{ng} / \mu \mathrm{L})$ trypsin solution at $4^{\circ} \mathrm{C}$ for $60 \mathrm{~min}$ and then $30 \mu \mathrm{L}$ of $25 \mathrm{mM} \mathrm{NH}_{4} \mathrm{HCO}_{3}$ buffer was added and incubated at $37^{\circ} \mathrm{C}$ overnight. The supernatant was directly applied onto the sample plate for MALDI-TOF MS analysis. MALDI-TOF/TOF MS analysis was performed using a Bruker Autoflex Speed MALDI-TOF/TOF MS (Bruker Daltonics, Bremen, Germany) operated in the reflector mode for MALDI-TOF peptide mass fingerprint (PMF) followed by LIFT mode for tandem MS in a fully automated approach using the FlexControl ${ }^{\mathrm{TM}}$ software. Samples were analyzed by one PMF from MALDI-TOF, followed by additional LIFT-TOF-TOF MS/MS analysis of the top 7-10 highest intensity peptides. Data were accumulated from 1000 consecutive laser shots to produce PMF followed by 200 precursor ion scans and $2000 \mathrm{MS} / \mathrm{MS}$ spectra in LIFT mode. Calibration for PMF samples (digests) was performed both externally on an adjacent spot for each sample using a mixture of nine peptides ranging from $\mathrm{m} / \mathrm{z} 757.40$ to 3147.47. The $\mathrm{m} / \mathrm{z}$ range was $700-4000$ for PMF and dynamically adjusted for each ion in MS/MS. PMF and MS/MS spectra were interpreted primarily with the FlexAnalysis ${ }^{\mathrm{TM}}$ software (Bruker Daltonics). Signal-to-noise ratio threshold was set to three. Blank gel was used as blank control through the entire steps.

\section{Database Searching of MALDI-TOF/TOF MS}

For protein identification, database searches were carried out with MASCOT version 2.3 (Matrix Science, London, UK) against NCBInr database and were performed via BioTools 3.2 software (Bruker Daltonics). Species were restricted to bacteria (Eubacteria). The parameters used are: a specified trypsin enzymatic cleavage with one possible missed cleavage, monoisotopic masses, a mass tolerance of $\pm 50 \mathrm{ppm}$ for the parent ion and $\pm 0.7 \mathrm{Da}$ for the fragment ion. Carbamidomethylation of cysteine residues as fixed modification, oxidation of methionine as differential modifications. The protein identification was inferred from peptide-spectrum matches by assembling the identified peptide sequences into proteins.

\section{Results}

\section{Antimicrobial Susceptibility and DNA Sequence Analysis}

Minimum inhibitory concentrations and $\beta$-lactamase genes of the 18 strains were listed in Table $\mathbf{1}$. In addition to two susceptible 
clinical isolates and two ATCC strains, the other 14 isolates showed reduced susceptibility or resistance to carbapenems. $\beta$-lactamase genes were detected in all the 14 strains, five of them produced KPC-2-type carbapenemase, and two of them produced IMP-4-type carbapenemase (Table 1).

\section{Sodium Dodecylsulfate-Polyacrylamide Gel Electrophoresis}

The arrows observed in the ATCC strains between molecular weight of $36-48 \mathrm{kDa}$ were assigned to $48 \mathrm{kDa}-\mathrm{LamB}, 44 \mathrm{kDa}$ putative OMP, $42 \mathrm{kDa}-\mathrm{OmpK} 36$, and $40 \mathrm{kDa}-\mathrm{OmpA}$ for K. pneumoniae isolates and $41 \mathrm{kDa}-\mathrm{OmpC}, 40 \mathrm{kDa}-\mathrm{OmpF}$, and $38 \mathrm{kDa}-O m p A$ for E. coli isolates, respectively (Figure 1). Fortyone $\mathrm{kDa}-\mathrm{OmpC}$ and $40 \mathrm{kDa}-\mathrm{OmpF}$ bands in E. coli ATCC 25922 were in close proximity and were difficult to be distinguished (Figure 1). OmpK35 was not found in the current study. K. pneumoniae KP1-KP3 and KP6 failed to express OmpK36. E. coli EC1-EC3, EC6-EC8 failed to express OmpC and OmpF; E. coli EC4 and EC5 failed to express OmpF.

\section{MALDI-TOF MS Analysis}

Six major peaks with $\mathrm{m} / \mathrm{z}$ of ca. 17,500, 18,500, 19,000, 35,000, 37,000 , and 38,000, respectively, were detected in E. coli ATCC 25922 which expressed porins as analyzed by SDS-PAGE. The ca. $-17,500,18,500$, and $19,000-\mathrm{m} / \mathrm{z}$ peaks $(\mathrm{M}+2 \mathrm{H})^{2+}$ observed in some strains were considered to be the multiple charged state of the ca. $-35,000,37,000$, and $38,000-\mathrm{m} / \mathrm{z}$ peaks. The ca. $-35,000$, 37,000 , and $38,000-\mathrm{m} / \mathrm{z}$ peaks were presumed to be OmpA, OmpF, and OmpC with $\sim 38,40$, and $41 \mathrm{kDa}$ in SDS-PAGE gel, respectively. For the E. coli EC1-EC3, EC6-EC8 isolates which failed to express $\mathrm{OmpC}$ and $\mathrm{OmpF}$ porins, the peaks of ca. $-37,000$ and $38,000-\mathrm{m} / \mathrm{z}$ disappeared along with the $(\mathrm{M}+2 \mathrm{H})^{2+}$ peaks ca. $-18,500$ and $19,000-\mathrm{m} / \mathrm{z}$. Similarly, the ca. $-37,000-\mathrm{m} / \mathrm{z}$ disappeared along with the $(\mathrm{M}+2 \mathrm{H})^{2+}$ peak ca. $-18,500-\mathrm{m} / \mathrm{z}$ mass spectra in the OmpF-deficient E. coli EC4 and EC5 isolates (Figure 2).

As with E. coli isolates, six major peaks with $\mathrm{m} / \mathrm{z}$ of ca. $18,000,18,500,19,000,36,000,37,000$, and 38,600 , respectively, were observed in $K$. pneumoniae ATCC 13883, KP4, KP5, and KP7. The ca. $-18,000,18,500$, and $19,300-\mathrm{m} / \mathrm{z}$ peaks $(\mathrm{M}+2 \mathrm{H})^{2+}$ observed in some strains were considered to be the multiple charged state of the ca. $-36,000,37,000$, and $38,600-\mathrm{m} / \mathrm{z}$ peaks. The ca. $-36,000$ and $38,600-\mathrm{m} / \mathrm{z}$ peaks were presumed to be OmpA and OmpK36 with $\sim 40$ and $42 \mathrm{kDa}$ in SDS-PAGE, respectively. The ca. $-37,000-\mathrm{m} / \mathrm{z}$ peak was presumed to be OmpK35 which was not detected by SDS-PAGE. The intensity of signal of the ca. $-37,000-\mathrm{m} / \mathrm{z}$ peak was much lower than the ca. $-36,000$ and 38,600 $\mathrm{m} / \mathrm{z}$ peaks. For the OmpK36-deficient isolates, the peak of ca. $-38,600-\mathrm{m} / \mathrm{z}$ disappeared along with the $(\mathrm{M}+2 \mathrm{H})^{2+}$ peak ca. $-19,300-\mathrm{m} / \mathrm{z}$. Mass spectrum of $K$. pneumoniae ATCC 13883 (similar with KP5 and KP7), KP1 (similar with KP3 and KP6), KP2 and KP4 were displayed in Figure 3. Fortyeight $\mathrm{kDa}-\mathrm{LamB}$ and $44 \mathrm{kDa}$-putative OMP observed in SDSPAGE of some strains were not detected in MALDI-TOF MS, maybe due to the large molecular weight or low intensity of signal.

\section{MALDI-TOF/TOF MS Analysis}

Twelve protein bands were successfully identified by MALDITOF/TOF MS after in-gel tryptic digestion. These protein bands represented for well-known proteins such as OmpA, OmpC, OmpF, OmpK36, and LamB. Bands 2, 3, 8, 9, 11, 12 were identified by peptide mass sequence analysis as OmpA; Band 1 was identified as a mixture of OmpC and OmpF, and Band 4 was identified as OmpC. Band 7 matched well to OmpK36 (score 441, sequence coverage 24\%). However, bands 6 and 10 which might be mistaken for OmpK35 were identified as putative OMP. Mass spectrum of tryptic-digested and MALDI-TOF/TOF MS analyzed OmpA, OmpC, OmpK36 and putative OMP were showed in Supplementary Figures S1S8. None of the OmpK35 bands were found in the current study. The names of the proteins, MASCOT scores, peptide sequences and the specific correspondence between SDS-PAGE, MALDI-TOF MS and MALDI-TOF/TOF MS were indicated in Table 2 .

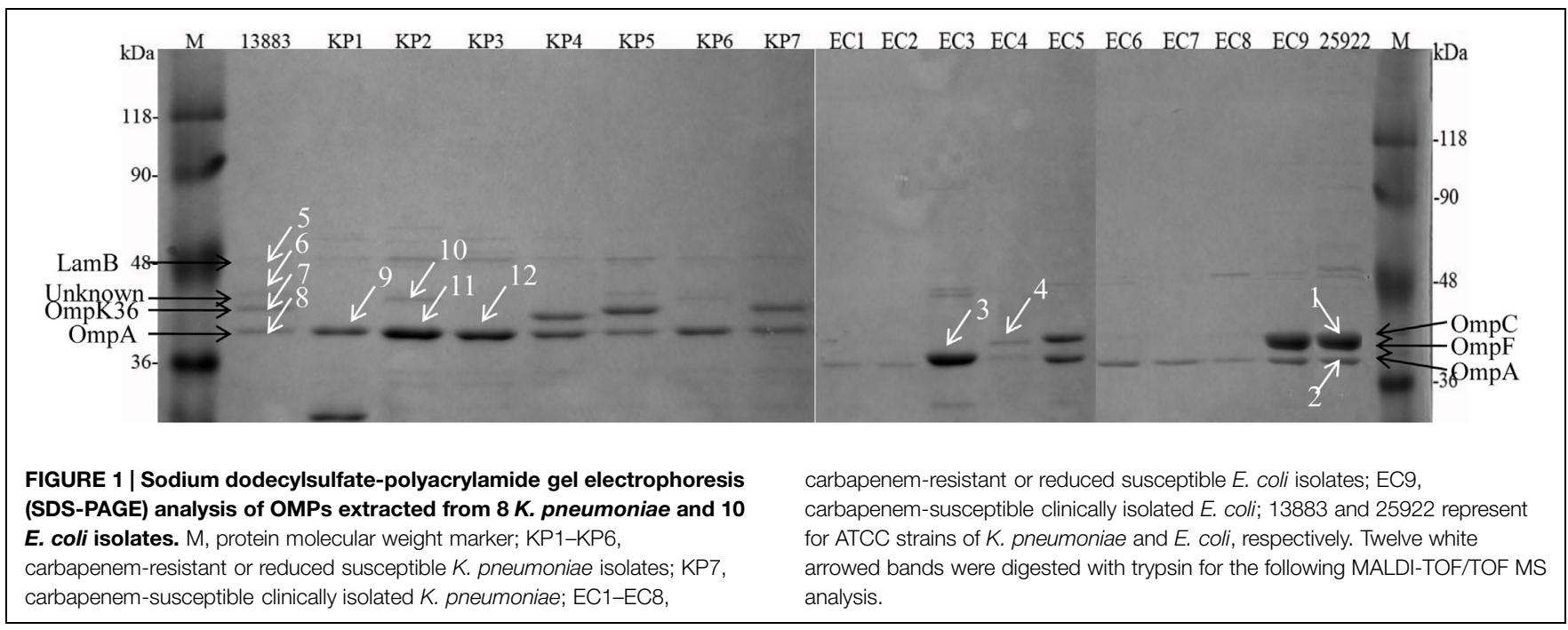




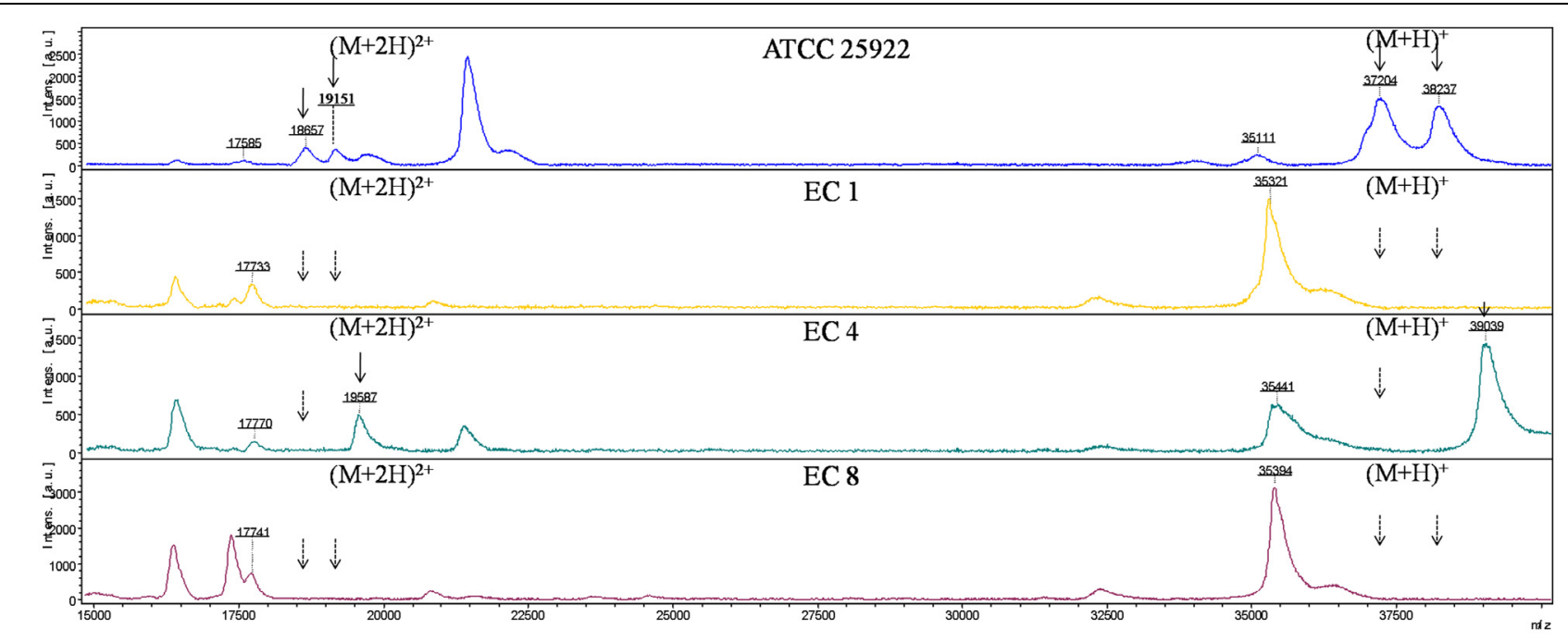

FIGURE 2 | MALDI-TOF MS analysis of $E$. coli isolates. $x$ axis, mass per charge in Daltons ( $\mathrm{m} / \mathrm{z}, \mathrm{Da})$; $\mathrm{y}$ axis, absolute intensity of signal. The solid arrows represent peaks with approximately 37,000and $38,000-\mathrm{m} / \mathrm{z}$ corresponding to $\mathrm{OmpC}$ and $\mathrm{OmpF}$, and their corresponding peaks of $18,500-$ and $19,000-\mathrm{m} / \mathrm{z}$ with $(\mathrm{M}+2 \mathrm{H})^{2+}$. The dotted arrows indicate the loss of 18,500-, 19,000-, 37,000- and $38,000-\mathrm{m} / \mathrm{z}$ peaks. $35,000-\mathrm{m} / \mathrm{z}$ peak was detected in all the strains representing for OmpA.

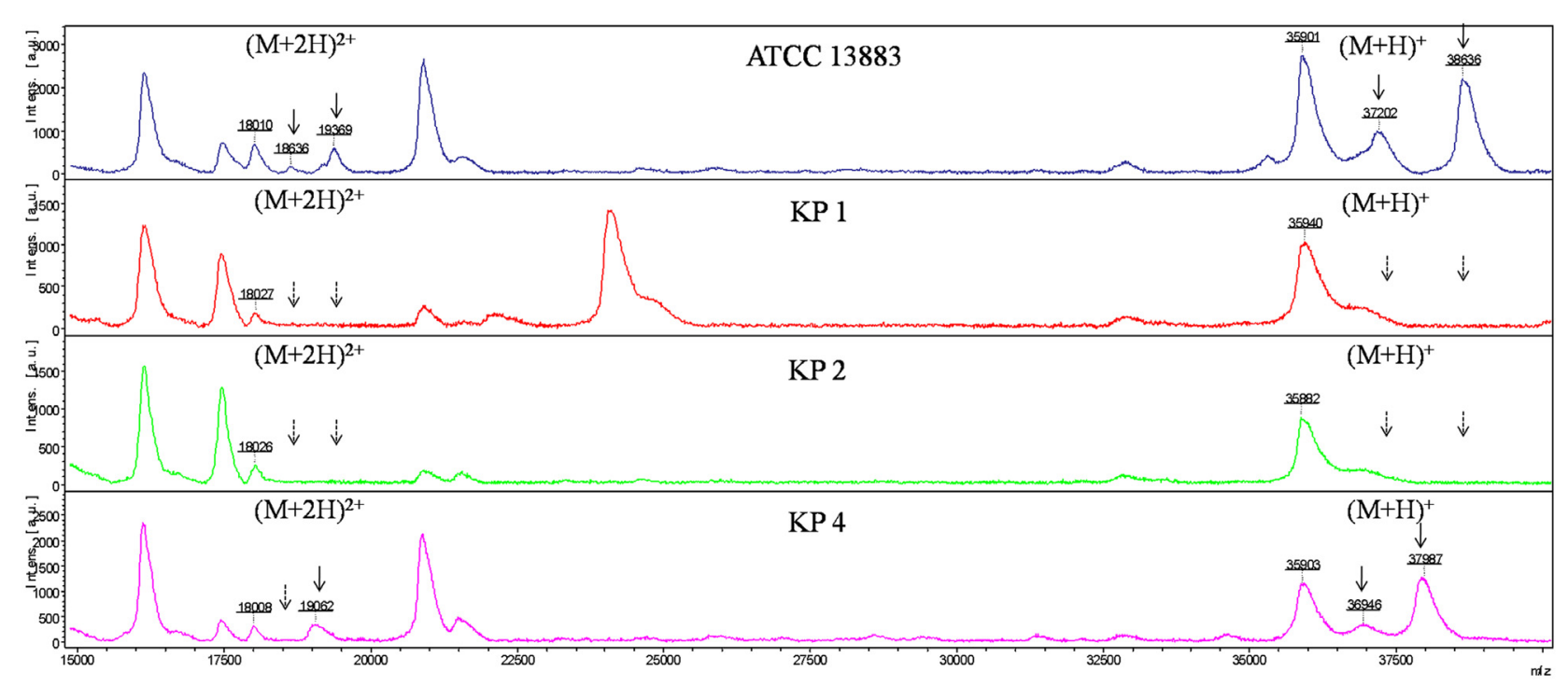

FIGURE 3 | MALDI-TOF MS analysis of $K$. pneumoniae isolates. $x$ axis, mass per charge in Daltons $(\mathrm{m} / \mathrm{z}, \mathrm{Da})$; y axis, absolute intensity of signal. The solid arrows represent peaks with approximately 37,000 - and 38,600-m/z corresponding to OmpK35 and OmpK36, and their corresponding peaks of 18,500- and 19,300-m/z with $(\mathrm{M}+2 \mathrm{H})^{2+}$. The dotted arrows indicate the loss of 18,500-, 19,300-, 37,000-, and $38,600-\mathrm{m} / \mathrm{z}$ peaks. $36,000-\mathrm{m} / \mathrm{z}$ peak was detected in all the strains representing for OmpA.

\section{Discussion}

MALDI-TOF MS is an emerging method where material is ionized in a high vacuum chamber and accelerated in an electric field. The fragment size $(\mathrm{m} / \mathrm{z})$ can be inferred from the time of flight of the ionized fragments. In the last 2 years MALDI-TOF MS had became a rapid detection tool for carbapenemases and other $\beta$-lactamases, which has significantly reduced the detection time to about $4 \mathrm{~h}$ (Burckhardt and Zimmermann, 2011; Sparbier et al., 2012). We previously reported the rapid detection of porins by MALDI-TOF MS in K. pneumoniae isolates (Cai et al., 2012). Our current study extended the species to $E$. coli isolates and further confirmed the specific correspondences between SDSPAGE and MALDI-TOF MS.

Combining with SDS-PAGE, MALDI-TOF MS and MALDITOF/TOF MS, we obviously observed that the mass spectrum of ca. $35,000,37,000$, and $38,000-\mathrm{m} / \mathrm{z}$ peaks of E. coli ATCC 25922 corresponded to OmpA, OmpC, and $\mathrm{OmpF}$ with molecular 


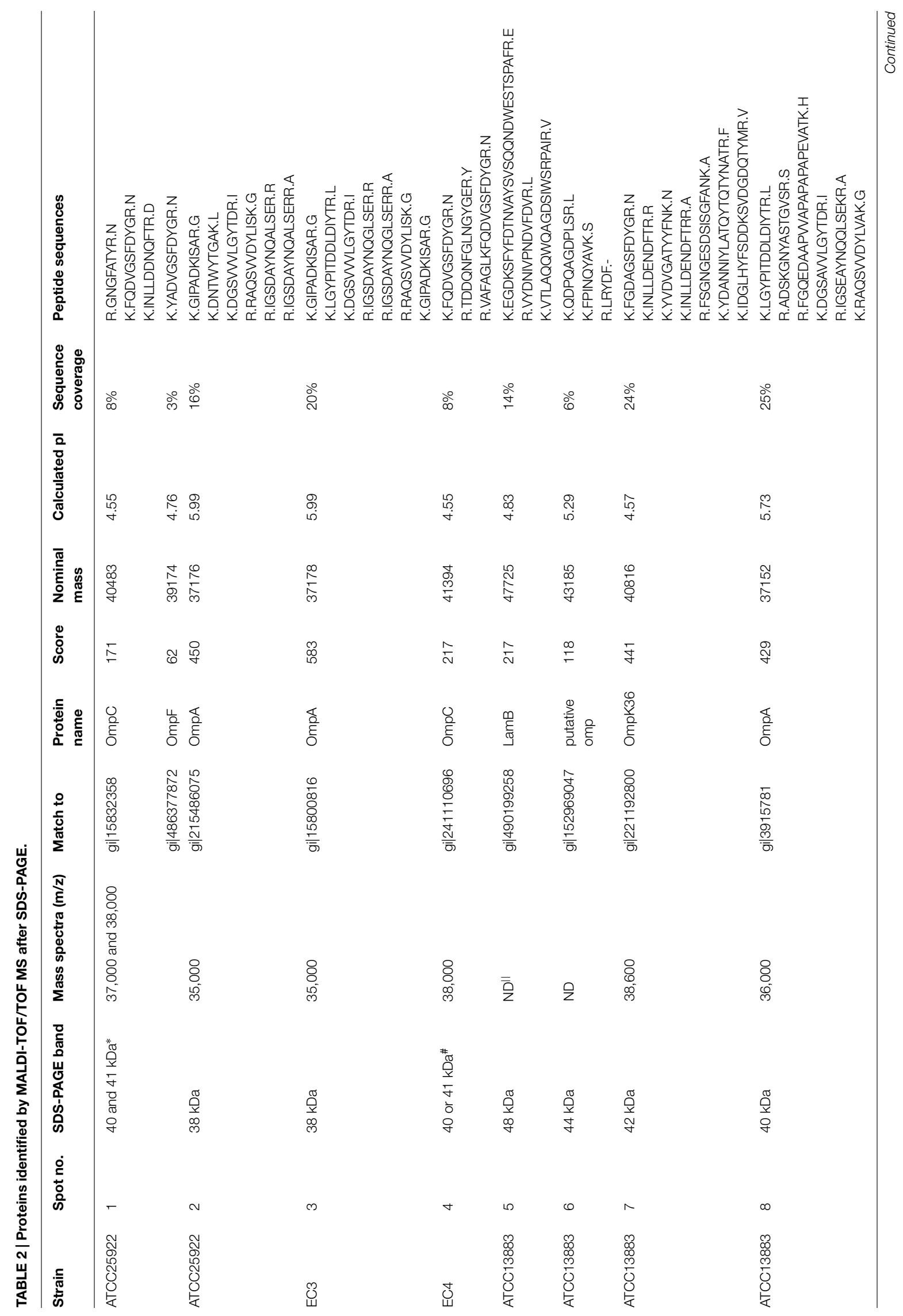




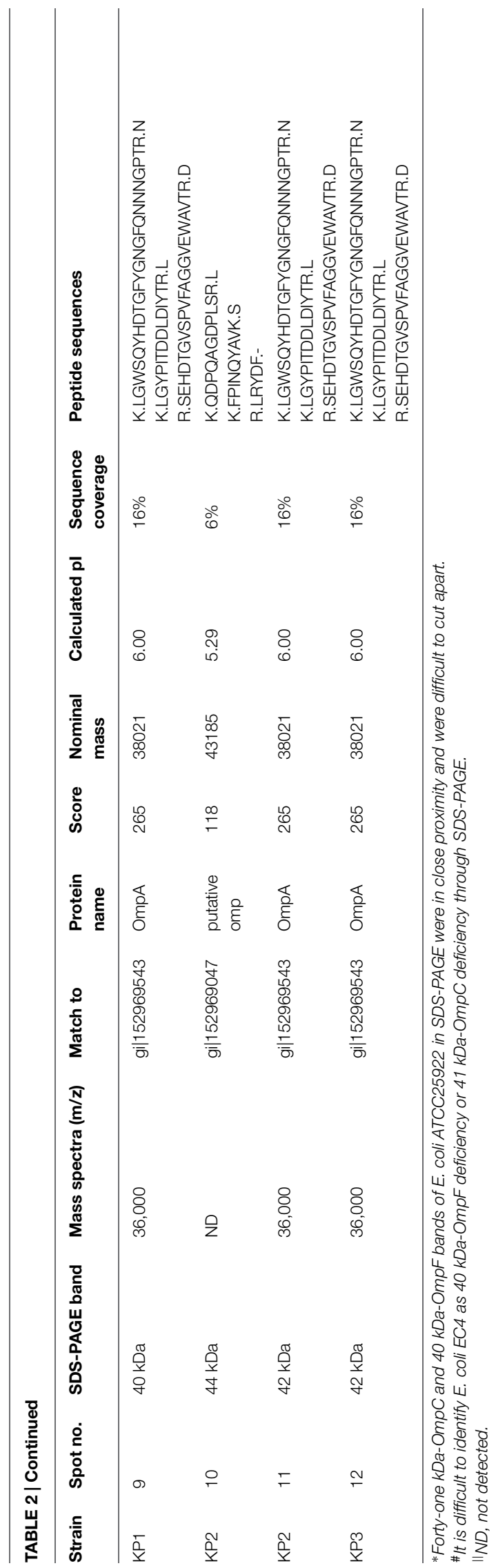

weight of ca. 38, 40, and $41 \mathrm{kDa}$ in SDS-PAGE, respectively. $\mathrm{OmpC}$ and $\mathrm{OmpF}$ are reported to be related to carbapenemresistance and $\mathrm{OmpF}$ migrates slightly faster than $\mathrm{OmpC}$ (Nikaido et al., 1983). They were difficult to be distinguished in the SDS-PAGE gel after a $2.5 \mathrm{~h}$ electrophoresis in the current study. Nevertheless, they could be clearly distinguished by MALDI-TOF MS through the $\mathrm{m} / \mathrm{z}$ of ca. 37,000 and 38,000. Meanwhile, it was hard to determine the E. coli EC4 and EC5 strains as OmpC-deficient or OmpF-deficient isolates through SDS-PAGE. However, via the mass spectra of E. coli EC4 and EC5, it was unambiguous that the ca. $37,000-\mathrm{m} / \mathrm{z}$ peak corresponded to OmpF. Accordingly, we can draw the conclusion that E. coli EC4 and EC5 were OmpF-deficient isolates. It follows that the resolution of MALDI-TOF MS is much higher than SDSPAGE.

Porin OmpK36, whose amino acid sequence is very closer to that of E. coli OmpC porin and porin OmpK35 whose sequence is homologous to that of $E$. coli OmpF porin are the resistance-related porins in K. pneumoniae isolates (Alberti et al., 1995; Hernandezalles et al., 1995; Hernandez-Alles et al., 1999). The mass spectrum of ca. 36,000 and 38,600$\mathrm{m} / \mathrm{z}$ peaks of K. pneumoniae ATCC 13883 corresponded to OmpA and OmpK36 with molecular weight of ca. 40 and $42 \mathrm{kDa}$ in SDS-PAGE, respectively. Porin OmpK35 was not observed in the current SDS-PAGE, while a $37,000-\mathrm{m} / \mathrm{z}$ peak was found in $K$. pneumoniae ATCC 13883 between 36,000 and $38,600-\mathrm{m} / \mathrm{z}$ peaks which might be the characteristic peak of OmpK35 porin. Observation of $37,000-\mathrm{m} / \mathrm{z}$ peak mass spectrum in K. pneumoniae ATCC 13883, KP4, KP5, and KP7 isolates, which was not found in the corresponding bands in the SDS-PAGE probably predicted that OmpK35 expressed at low level and was unable to be detected or it was difficult to be separated from OmpK36 within $2.5 \mathrm{~h}$. The ESBLs-producing $K$. pneumoniae KP1, KP2, KP3, and KP6 were deficient in OmpK36 porin. Simultaneously, OmpK35 porin was not detected and the $37,000-\mathrm{m} / \mathrm{z}$ peak disappeared together with the $38,600-\mathrm{m} / \mathrm{z}$ peak which indicated that these isolates were deficient in both OmpK35 and OmpK36 porins. OmpK35 was not detected in all the isolates by SDS-PAGE, which result is in accordance with previous research which described that OmpK35 do not express or express at very low levels in the ESBLs-producing $K$. pneumoniae isolates and OmpK35 of 83\% (24/29) isolates are undetectable by SDS-PAGE (Hernandez-Alles et al., 1999). While it is still inexplicable why K. pneumoniae ATCC 13833 lacks OmpK35 band, further study was needed.

In addition, K. pneumoniae ATCC 13883 and KP2 expressed a $44 \mathrm{kDa}$ protein designated as a putative OMP. OmpK35 and OmpK36 migrates differently in various conditions. HernandezAlles et al. (1999) described that OmpK36 migrate faster than OmpK35. Therefore, the $44 \mathrm{kDa}$-putative OMP band might be easily mistaken for porin OmpK35 in the SDSPAGE gel, whereas the high resolution of MALDI-TOF MS make it easy to observe the $37,000-\mathrm{m} / \mathrm{z}$ peak which was presumed to be the characteristic peak of OmpK35. Therefore, we can determine whether porin OmpK35 is expressed or not by MALDI-TOF MS rather than by SDS-PAGE. 
The molecular weight of K. pneumoniae isolates of OmpK36 ( 42 vs. $38 \mathrm{kDa}$ and OmpA ( 40 vs. $32 \mathrm{kDa}$ ) in the present study were slightly different with our previous reports (Cai et al., 2008; Wang et al., 2009). This phenomenon might probably be due to the difference of apparent molecular weight in the SDSPAGE. Apparent molecular weight is normally different from the amino acids-based theoretical molecular weight. The apparent molecular weight is easily affected by many factors such as posttranslational modification of proteins, electrophoresis conditions $(\mathrm{PH}$, salt concentration) and the properties of proteins. All these factors might result in the discrepancy of apparent molecular weight with the theoretical molecular weight.

Moreover, Cornaglia et al. (1992) indicated that loss in either OmpC or OmpF did influence the susceptibility, although both were less important than the combined OmpC and OmpF deficiency. Meanwhile, Oteo et al. (2008) found that loss of OmpF did not affect the carbapenem susceptibility. The OmpF-deficient E. coli EC4 and EC5 isolates in present study produces KPCtype carbapenemase and CTX-M-type ESBLs, thus it is difficult to distinguish which factor(s) have resulted in the carbapenem resistance (production of carbapenemases or combination of high-level production of ESBLs together with porin loss or both). Whether porin OmpF played an important role in the carbapenem susceptibility requires further investigations.

The signal intensity of the ca. $-37,000-\mathrm{m} / \mathrm{z}$ peak in carbapenem-susceptible strains was much lower than the ca. $-36,000$ and $38,600-\mathrm{m} / \mathrm{z}$ peaks. In accordance with the mass spectrum, the high intensity ca. $-36,000$ and $38,600-\mathrm{m} / \mathrm{z}$ peaks

\section{References}

Alberti, S., Rodriquez-Quinones, F., Schirmer, T., Rummel, G., Tomas, J. M., Rosenbusch, J. P., et al. (1995). A porin from Klebsiella pneumoniae: sequence homology, three-dimensional model, and complement binding. Infect. Immun. 63, 903-910.

Baldwin, C. M., Lyseng-Williamson, K. A., and Keam, S. J. (2008). Meropenem: a review of its use in the treatment of serious bacterial infections. Drugs 68, 803-838. doi: 10.2165/00003495-200868060-00006

Bizzini, A., and Greub, G. (2010). Matrix-assisted laser desorption ionization timeof-flight mass spectrometry, a revolution in clinical microbial identification. Clin. Microbiol. Infect. 16, 1614-1619. doi: 10.1111/j.1469-0691.2010.03311.x

Burckhardt, I., and Zimmermann, S. (2011). Using matrix-assisted laser desorption ionization-time of flight mass spectrometry to detect carbapenem resistance within 1 to 2.5 hours. J. Clin. Microbiol. 49, 3321-3324. doi: 10.1128/JCM.00287-11

Cai, J. C., Hu, Y. Y., Zhang, R., Zhou, H. W., and Chen, G. X. (2012). Detection of OmpK36 porin loss in Klebsiella spp. by matrix-assisted laser desorption ionization-time of flight mass spectrometry. J. Clin. Microbiol. 50, 2179-2182. doi: 10.1128/JCM.00503-12

Cai, J. C., Zhang, R., Hu, Y. Y., Zhou, H. W., and Chen, G. X. (2014). Emergence of Escherichia coli sequence type 131 isolates producing KPC-2 carbapenemase in China. Antimicrob. Agents Chemother. 58, 1146-1152. doi: 10.1128/AAC.00912-13

Cai, J. C., Zhou, H. W., Zhang, R., and Chen, G. X. (2008). Emergence of Serratia marcescens, Klebsiella pneumoniae, and Escherichia coli Isolates possessing the plasmid-mediated carbapenem-hydrolyzing beta-lactamase KPC-2 in intensive care units of a Chinese hospital. Antimicrob. Agents Chemother. 52, 2014-2018. doi: 10.1128/AAC.01539-07

CLSI. (2013). Performance Standards for Antimicrobial Susceptibility Testing; Twenty-Third Informational Supplement. CLSI Document M100-S23. Wayne, PA: Clinical and Laboratory Standards Institute. corresponding to OmpA and OmpK36 were able to be observed in SDS-PAGE gel, while the low intensity ca. $-37,000-\mathrm{m} / \mathrm{z}$ peak corresponding to OmpK35 was undetectable by SDS-PAGE. Hence, we can presume that the intensity of signal might indirectly reflect the protein expression, which might expand the application of MALDI-TOF MS to assess the relative expression level of OMPs. Nevertheless, further study was needed to prove this point.

In summary, compared to SDS-PAGE, MALDI-TOF MS was able to rapidly identify the porin-deficient strains within half an hour. This method possesses better sensitivity, low cost, and is easier to operate and had less interference, therefore, it is suitable for application in general clinical laboratories.

\section{Acknowledgments}

We sincerely thank Dr. Zhaoyang Liu of Bruker (Beijing) Scientific Technology Co. Ltd. for the technical support of MALDI-TOF/TOF MS.

\section{Supplementary Material}

The Supplementary Material for this article can be found online at: http://journal.frontiersin.org/article/10.3389/fmicb. 2015.00784

CLSI. (2014). Performance Standards for Antimicrobial Susceptibility Testing; Twenty-fourth Informational Supplement. CLSI Document M100-S24. Wayne, PA: Clinical and Laboratory Standards Institute.

Cornaglia, G., Guan, L., Fontana, R., and Satta, G. (1992). Diffusion of meropenem and imipenem through the outer membrane of Escherichia coli K-12 and correlation with their antibacterial activities. Antimicrob. Agents Chemother. 36, 1902-1908. doi: 10.1128/AAC.36.9.1902

Domenech-Sanchez, A., Martinez-Martinez, L., Hernandez-Alles, S., Del Carmen Conejo, M., Pascual, A., Tomas, J. M., et al. (2003). Role of Klebsiella pneumoniae OmpK35 porin in antimicrobial resistance. Antimicrob. Agents Chemother. 47, 3332-3335. doi: 10.1128/AAC.47.10.3332-33 35.2003

Edwards-Jones, V., Claydon, M. A., Evason, D. J., Walker, J., Fox, A. J., and Gordon, D. B. (2000). Rapid discrimination between methicillin-sensitive and methicillin-resistant Staphylococcus aureus by intact cell mass spectrometry. J. Med. Microbiol. 49, 295-300.

Griffin, P. M., Price, G. R., Schooneveldt, J. M., Schlebusch, S., Tilse, M. H., Urbanski, T., et al. (2012). Use of matrix-assisted laser desorption ionizationtime of flight mass spectrometry to identify vancomycin-resistant enterococci and investigate the epidemiology of an outbreak. J. Clin. Microbiol. 50, 29182931. doi: 10.1128/JCM.01000-12

Hernandez-Alles, S., Alberti, S., Alvarez, D., Domenech-Sanchez, A., MartinezMartinez, L., Gil, J., et al. (1999). Porin expression in clinical isolates of Klebsiella pneumoniae. Microbiology 145(Pt 3), 673-679. doi: 10.1099/13500872-1453-673

Hernandezalles, S., Alberti, S., Rubires, X., Merino, S., Tomas, J. M., and Benedi, V. J. (1995). Isolation of Fc3-11, a bacteriophage specific for the Klebsiella pneumoniae porin ompk36, and its use for the isolation of porin-deficient mutants. Can. J. Microbiol. 41, 399-406. doi: 10.1139/ m95-053

Hrabak, J., Walkova, R., Studentova, V., Chudackova, E., and Bergerova, T. (2011). Carbapenemase activity detection by matrix-assisted laser desorption 
ionization-time of flight mass spectrometry. J. Clin. Microbiol. 49, 3222-3227. doi: 10.1128/JCM.00984-11

Ikryannikova, L. N., Afanas'ev, M. V., Akopian, T. A., Il'ina, E. N., Kuz'min, A. V., Larionova, E. E., et al. (2007). Mass-spectrometry based minisequencing method for the rapid detection of drug resistance in Mycobacterium tuberculosis. J. Microbiol. Methods 70, 395-405. doi: 10.1016/j.mimet.2007.05.015

Liu, H., Yang, L., Khainovski, N., Dong, M., Hall, S. C., Fisher, S. J., et al. (2011). Automated iterative MS/MS acquisition: a tool for improving efficiency of protein identification using a LC-MALDI MS workflow. Anal. Chem. 83, 6286-6293. doi: 10.1021/ac200911v

Liu, Y. F., Yan, J. J., Ko, W. C., Tsai, S. H., and Wu, J. J. (2008). Characterization of carbapenem-non-susceptible Escherichia coli isolates from a university hospital in Taiwan. J. Antimicrob. Chemother. 61, 1020-1023. doi: 10.1093/jac/ dkn049

Nagaraj, S., Chandran, S. P., Shamanna, P., and Macaden, R. (2012). Carbapenem resistance among Escherichia coli and Klebsiella pneumoniae in a tertiary care hospital in south India. Indian J. Med. Microbiol. 30, 93-95. doi: 10.4103/02550857.93054

Nikaido, H., Rosenberg, E. Y., and Foulds, J. (1983). Porin channels in Escherichia coli: studies with beta-lactams in intact cells. J. Bacteriol. 153, 232-240.

Oteo, J., Delgado-Iribarren, A., Vega, D., Bautista, V., Rodriguez, M. C., Velasco, M., et al. (2008). Emergence of imipenem resistance in clinical Escherichia coli during therapy. Int. J. Antimicrob. Agents 32, 534-537. doi: 10.1016/j.ijantimicag.2008.06.012

Papp-Wallace, K. M., Endimiani, A., Taracila, M. A., and Bonomo, R. A. (2011). Carbapenems: past, present, and future. Antimicrob. Agents Chemother. 55, 4943-4960. doi: 10.1128/AAC.00296-11

Perez-Perez, F. J., and Hanson, N. D. (2002). Detection of plasmid-mediated AmpC beta-lactamase genes in clinical isolates by using multiplex PCR. J. Clin. Microbiol. 40, 2153-2162. doi: 10.1128/JCM.40.6.2153-2162.2002

Prajanban, B. O., Shawsuan, L., Daduang, S., Kommanee, J., Roytrakul, S., Dhiravisit, A., et al. (2012). Identification of five reptile egg whites protein using MALDI-TOF mass spectrometry and LC/MS-MS analysis. J. Proteomics 75, 1940-1959. doi: 10.1016/j.jprot.2012.01.004

Queenan, A. M., and Bush, K. (2007). Carbapenemases: the versatile betalactamases. Clin. Microbiol. Rev. 20, 440-458. doi: 10.1128/CMR.00001-07
Sparbier, K., Schubert, S., Weller, U., Boogen, C., and Kostrzewa, M. (2012) Matrix-assisted laser desorption ionization-time of flight mass spectrometrybased functional assay for rapid detection of resistance against betalactam antibiotics. J. Clin. Microbiol. 50, 927-937. doi: 10.1128/JCM. 05737-11

Wang, X. D., Cai, J. C., Zhou, H. W., Zhang, R., and Chen, G. X. (2009). Reduced susceptibility to carbapenems in Klebsiella pneumoniae clinical isolates associated with plasmid-mediated beta-lactamase production and OmpK36 porin deficiency. J. Med. Microbiol. 58, 1196-1202. doi: 10.1099/jmm.0. 008094-0

Wieser, A., Schneider, L., Jung, J., and Schubert, S. (2012). MALDI-TOF MS in microbiological diagnostics-identification of microorganisms and beyond (mini review). Appl. Microbiol. Biotechnol. 93, 965-974. doi: 10.1007/s00253011-3783-4

Yigit, H., Queenan, A. M., Anderson, G. J., Domenech-Sanchez, A., Biddle, J. W., Steward, C. D., et al. (2001). Novel carbapenem-hydrolyzing betalactamase, KPC-1, from a carbapenem-resistant strain of Klebsiella pneumoniae. Antimicrob. Agents Chemother. 45, 1151-1161. doi: 10.1128/AAC.45.4.11511161.2001

Yu, Y., Ji, S., Chen, Y., Zhou, W., Wei, Z., Li, L., et al. (2007). Resistance of strains producing extended-spectrum beta-lactamases and genotype distribution in China. J. Infect. 54, 53-57. doi: 10.1016/j.jinf.2006.01.014

Zhang, R., Hu, Y. Y., Yang, X. F., Gu, D. X., Zhou, H. W., Hu, Q. F., et al. (2013). Emergence of NDM-producing non-baumannii Acinetobacter spp. isolated from China. Eur. J. Clin. Microbiol. Infect. Dis. 33, 853-860. doi: 10.1007/s10096-013-2024-4

Conflict of Interest Statement: The authors declare that the research was conducted in the absence of any commercial or financial relationships that could be construed as a potential conflict of interest.

Copyright (C) $2015 \mathrm{Hu}$, Cai, Zhou, Zhang and Chen. This is an open-access article distributed under the terms of the Creative Commons Attribution License (CC BY). The use, distribution or reproduction in other forums is permitted, provided the original author(s) or licensor are credited and that the original publication in this journal is cited, in accordance with accepted academic practice. No use, distribution or reproduction is permitted which does not comply with these terms. 